4 Shoji T, Hanaoka N, Wada H, et al. Balloon angioplasty for pulmonary artery stenosis after lung transplantation. Eur J Cardiothorac Surg 2008; 34: 693-694.

5 Waurick PE, Kleber FX, Ewert R, et al. Pulmonary artery stenosis 5 years after single lung transplantation in primary pulmonary hypertension. J Heart Lung Transplant 1999; 18: 1243-1245.

6 Doyle T, Loyd J, Robbins IM. Percutaneous pulmonary artery and vein stenting. Am J Respir Crit Care Med 2001; 164: 657-660.
7 Gaubert J, Moulin G, Thomas P, et al. Anastomotic stenosis of the left pulmonary artery after lung transplantation: treatment by percutaneous placement of an endoprosthesis. AJR Am J Roentgenol 1993; 161: 947-949.

8 Berger H, Steiner W, Schmidt D, et al. Stent-angioplasty of an anastomotic stenosis of the pulmonary artery after lung transplantation. Eur J Cardiothorac Surg 1994; 8: 103-105.

DOI: $10.1183 / 09059180.00009610$

\title{
Tobacco smoking: why do physicians not make diagnoses?
}

\section{To the Editor:}

Tobacco smoking is recognised as the single greatest cause of avoidable morbidity and mortality in developed countries. An enormous amount of scientific evidence to support this statement has been collected in the past 60 yrs. Searching for "tobacco smoking and diseases" through PubMed provides results for 62,976 papers published since 1950. Notwithstanding this, physicians often forget that tobacco smoking, in addition to being a cause of many different diseases, is a disease itself.

In 1980, for the first time in the history of disease classification, the diagnosis of tobacco dependence was included in the third edition of the Diagnostic and Statistical Manual of Mental Disorders (DSM).

In 1988, the US Surgeon General issued a seminal report entitled "Nicotine Addiction", in which it was clearly stated that cigarettes were addictive, nicotine was the drug in tobacco that causes addiction, and tobacco addiction was similar to other substance addictions, such as to heroin or cocaine.

In 1992, the World Health Organization (WHO) included tobacco smoking under the "Mental and Behavioural Disorders" and, in the International Classification of Diseases 10, tobacco dependence has been defined as "a cluster of behavioural, cognitive and physiological phenomena that develop after repeated use".

In 1994, the DSM IV referred to nicotine dependence and nicotine withdrawal as psychiatric disorders, and the DSM IV Text Revised (DSM IV-TR) introduced the section of nicotine use disorders, including nicotine dependence, nicotine-induced disorders and nicotine-related disorders not otherwise specified.

In 2007, the European Respiratory Society (ERS) Task Force on smoking cessation in patients with respiratory diseases stated that tobacco smoking/nicotine addiction can be regarded as a chronic, recurrent disease; that dependence on tobacco is a complex behaviour, with both environmental and genetic influences; and that nicotine is the main component in cigarettes that contributes to addiction, although psychological factors and habituation also play a role [1].

Over time, tobacco smoking has been demonstrated to be a disease associated with younger age, lower income, reduced educational achievement and disadvantaged neighbourhood environment [2]. Moreover, risk factors increasing susceptibility to tobacco dependence have been well documented: starting smoking early in adolescence; comorbidity with mental illnesses or substance abuse disorders; and genetic-based rapid metabolism of nicotine [3].

If tobacco smoking has been definitively defined as a disease, why do doctors not usually make this diagnosis?

Different demographic or socio-economic conditions associated with the patient may reduce the probability that a smoker will be diagnosed. For example, patients of low socio-economic classes may have greater difficulty in accessing healthcare, even in some developed countries. However, most barriers in diagnosing tobacco dependence appear, surprisingly, to be related just to physicians. These can be summarised as follows.

1) Cultural heritage. Health personnel often use corroborated terms rather than the more new and appropriate ones. This cultural heritage seems to exist in different medical disciplines. However, for other pathological conditions, the use of an old term has not prevented the formulation the proper diagnosis. For instance, "absence seizure", introduced in 1981 by the Commission on Classification and Terminology of the International League against Epilepsy, is a diagnosis used also by those clinicians who still name the diseases as "petit mal seizures", a French term dating from the late 1700s.

2) Medical school training. In some countries (e.g. Italy, Spain, UK and Belgium), medical students are still trained to collect data on tobacco smoking (e.g. cigarettes smoked daily and packyears) in the physiological part of the case history. However, as a result of personal communications with other physicians, we note that in other countries (e.g. India and Croatia), tobacco dependence is correctly included among other concomitant diseases in the pathological part of the case history.

3) Reticence in recognising tobacco dependence as a relevant health problem. The health community seems to neglect tobacco dependence, not only when patients' health problems are considered, but also when their own health is considered. A high prevalence of smoking among physicians may explain this attitude. Although only 3\% of Canadian physicians [4], 4.4\% of ERS members [5], and $12 \%$ of Swiss primary care physicians currently smoke cigarettes [6], almost one-third (32.1\%) of French physicians [7] and $38.6 \%$ of Greek physicians are current smokers [8]. In Italy, the overall prevalence of smoking among hospital staff is $30.6 \%$ and among physicians is $21.2 \%$; moreover, only $58.7 \%$ of hospital health staff tackles the issue of smoking in 
any contact with patients [9]. In Switzerland, smokers did not receive additional advice to quit or help to quit smoking by their physicians over a period of 10 yrs [10].

The reticence in recognising tobacco dependence also seems to be widespread in clinical research. In a recent review, Cosci et al. [11] found that only a minority of studies on panic disorder and cigarette smoking referred to a formal diagnosis of tobacco dependence.

4) Formulation of a clinical diagnosis. This is probably only an apparent limitation, as it is not the only case of formulation of a clinical diagnosis in medicine. All psychiatric disorders are diagnosed using clinical criteria. For instance, according to the DSM IV-TR, major depression is diagnosed when at least five out of a set of nine symptoms are present for 2 weeks and determine a detriment of previous social/working functioning. Moreover, pulmonary physicians, internal medicine doctors and general practitioners usually use the clinical diagnosis of chronic bronchitis when the patient reports chronic or recurrent excessive mucous secretion in the bronchial tree, that is not attributable to other lung diseases and occurs on most days for at least 3 months in the year during a period of at least 2 yrs.

It should be noted that the clinical diagnosis of tobacco dependence is formulated using standardised instruments (e.g. WHO Composite International Diagnostic Interview version 1.1 [12]) and the severity of nicotine dependence is measured via validated scales (i.e. the Fagerstrom Test for Nicotine Dependence) [13].

Although there may be some limitations, they are not strong and plausible enough to justify such an attitude of underdiagnosing tobacco dependence.

All physicians should be encouraged to diagnose tobacco dependence, as this is a specific and frequent disorder transversal to all medical specialties. A clinical diagnosis of tobacco dependence may be easily made by asking standardised questions in a few minutes, in the same way as it is possible to diagnose major depression or chronic bronchitis.

Thinking in terms of tobacco dependence instead of tobacco smoking might facilitate physicians in considering current smokers as patients needing of treatment, and former smokers as patients with a lifetime disorder at risk of relapse who need surveillance.

\section{F. Cosci* , F. Pistelli ${ }^{\#}$ and L. Carrozzi ${ }^{\#}$}

*Dept of Psychology, University of Florence, Florence, and

"Pulmonary and Respiratory Pathophysiology Unit, Cardiothoracic Dept, University Hospital of Pisa, Pisa, Italy.
Correspondence: F. Cosci, Dept of Psychology, University of Florence, Via di San Salvi, 12 Padiglione 26, 50135 Florence, Italy. E-mail: fiammetta.cosci@unifi.it

Statement of Interest: None declared.

Provenance: Submitted article, peer reviewed.

Acknowledgements: F. Cosci wishes to thank the colleagues of the International College in Affective NeuroScience (ICANS) for their personal communications.

\section{REFERENCES}

1 Tønnesen P, Carrozzi L, Fagerström KO, et al. Smoking cessation in patients with respiratory diseases: a high priority, integral component of therapy. Eur Respir J 2007; 29: 390-417.

2 Bergen AW, Caporaso N. Cigarette smoking. J Natl Cancer Inst 1999; 91: 1365-1375.

3 Benowitz NL. Nicotine addiction. $N$ Engl J Med 2010; 362: 2295-2303.

4 Frank E, Segura C. Health practices of Canadian physicians. Can Fam Physician 2009; 55: 810-811.

5 Clancy L, Ward B, Kabir Z. Active and passive smoking in the ERS membership. Eur Respir J 2010; 36: Suppl. 54, 940s.

6 Sebo P, Bouvier Gallacchi M, Goehring C, et al. Use of tobacco and alcohol by Swiss primary care physicians: a cross-sectional survey. BMC Public Health 2007; 7: 5.

7 Josseran L, King G, Guilbert P, et al. Smoking by French general practitioners: behaviour, attitudes and practice. Eur J Public Health 2005; 15: 33-38.

8 Sotiropoulos A, Gikas A, Spanou E, et al. Smoking habits and associated factors among Greek physicians. Public Health 2007; 121: 333-340.

9 Sabato E, Carrozzi L, Nardini S, et al. Progetto Obiettivo AIPO "Ospedali senza fumo": risultati della III fase [AIPO task force "Smoke Free Hospitals": results from phase III]. Rassegna di Patologia dell'Apparato Respiratorio 2004; 19: 247-261.

10 Etter JF. Smoking prevalence, cigarette consumption and advice received from physicians: change between 1996 and 2006 in Geneva, Switzerland. Addict Behav 2010; 35: 355-358.

11 Cosci F, Knuts IJ, Abrams K, et al. Cigarette smoking and panic: a critical review of the literature. J Clin Psychiatry 2010; 71: 606-615

12 World Health Organization. Composite International Diagnostic Interview (Version 1.1). Washington, American Psychiatric Press, 1993.

13 Heatherton TF, Kozlowski LT, Frecker RC, et al. The Fagerstrom Test for Nicotine Dependence: a revision of the Fagerstrom Tolerance Questionnaire. Br J Addict 1991; 86: 1119-1127.

DOI: $10.1183 / 09059180.00007210$ 